\title{
Presentation of a new Method for Determination of Diffusion Coefficients of Gaseous Pollutants in Cladding Wall Materials Indoors
}

\author{
J.J. Gonçalves ${ }^{1 \mathrm{a}}$, A.S. Santos ${ }^{1 \mathrm{~b}}$, G.V. Silva ${ }^{1 \mathrm{c}}$, E.O. Fernandes ${ }^{1 \mathrm{~d}}$ \\ ${ }^{1}$ IDMEC, Institute of Mechanical Engineering, University of Porto, Rua Dr. Roberto Frias, \\ 4200-465 Porto, Portugal \\ ajpg@fe.up.pt, basantos@fe.up.pt, cgvs@fe.up.pt, deof@fe.up.pt.
}

\begin{abstract}
Keywords: Diffusion coefficient, Mass diffusion, Building materials, Volatile organic compounds, Indoor air quality.
\end{abstract}

\begin{abstract}
Diffusion and partition coefficients are two key parameters of importance for the characterization of building materials as sources and sinks of air pollutants indoors. The number of coefficients for each pair 'chemical compound - material surface' available in the literature are still scarce and with a high discrepancy in the results obtained by different methods, even for the same method [1]. The objectives of this study were (1) to develop a simple and rapid experimental method for directly measuring the diffusion coefficient $(D)$, and (2) to perform determinations with both the new and the cup method comparing the results obtained. The new method is based on two existent methodologies, the passive sampling on Tenax TA and the dry cup method, proposed by Haghighat et al. [1] and also developed by this research team [2]. The experiments were carried for three different building materials (cork based material, gypsum board and PVC) and three different compounds (cyclohexane, n-octane and m-xylene). The discussion of the results obtained for $D$ by the two methods is presented in this paper. Observed differences were not of the same order of magnitude for the pairs compound/material studied, varying from the order of $10^{1}$ in the case of PVC to $10^{2}$ in the gypsum and in the cork. However some facts were the same: the gypsum board presents the higher values of $D$ and PVC the lower values of $D$ for the three compounds studied in both methods. One of the causes of these marked differences could be high difference of the concentration of the compound in the air between the methods, saturation value in cup method (dozens $\mathrm{g} / \mathrm{m}^{3}$ ) and low values typical from indoor environments (few $\mathrm{mg} / \mathrm{m}^{3}$ ) in the proposed method.
\end{abstract}

\section{Intoduction}

The quality of the indoor environment of non-industrial buildings has been receiving a growing interest from the scientific community due to the implications of the 'non quality' on the health and well being of the occupants. Many studies present construction and cladding materials as main sources of indoor air pollution, particularly of volatile organic compounds (VOC).

In this context the development of mathematical models for the prevision of the concentration of pollutants in real situations requires the previous study of the behaviour of the materials $v s$ pollutants to quantify the parameters involved, represented namely by the diffusion coefficients and partition coefficients [3]. The available data in the literature for those coefficients is still scarce and, in general, with highly dispersed values. The main objective of the present work was to develop a new method for determination of $D$. The values of $D$ were also determined by the cup method for comparison.

\section{Research methods}

Diffusion is the mode of mass transfer of a compound caused by a concentration gradient. Diffusion transport of gases in building materials involves a complex variety of processes including molecular, Knudsen and surface diffusion within the porous interstices [4]. Several experimental techniques have been used to estimate the diffusion coefficients. Three major methods for the 
measurement of diffusion coefficients of building materials have been used: the wet cup [5], the twin chamber [6], and the porosity test method [4].

Wet cup method._The simplest approach to measure the diffusion coefficient of building materials is the wet cup method $[5,7]$ and is based on Fick's $1^{\text {st }}$ law [8]. A thin plate of material (sample) is placed between two constant different concentrations sources (see model on Figure 1). After a time period, the concentrations in surfaces of the plate come into equilibrium with sources and a constant gradient of concentrations is established.
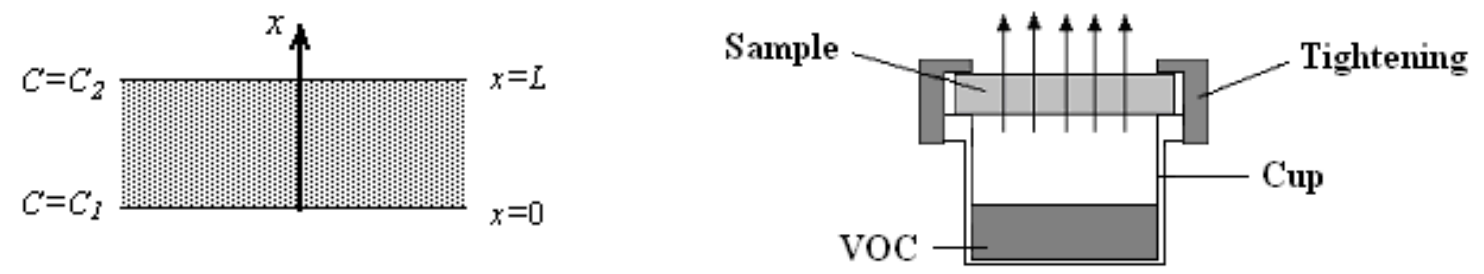

Figure 1. Steady one-dimensional Figure 2. Scheme of the cup used for diffusion tests. diffusion through a plate (model).

Under these conditions of diffusion, the mass flux, $J,\left(\mathrm{~kg} / \mathrm{m}^{2} . \mathrm{s}\right)$ of the diffusing substance can be expressed by:

$$
J=D \frac{C_{1}-C_{2}}{L}
$$

where $D$ is the effective diffusivity or diffusion coefficient $\left(\mathrm{m}^{2} / \mathrm{s}\right), C_{1}$ and $C_{2}$ are the surface concentrations $\left(\mathrm{kg} / \mathrm{m}^{3}\right)$, and $L$ is the thickness of the plate $(\mathrm{m})$. Eq. 1 suggests a method for the evaluation of the diffusion coefficient, since $J, C_{1}, C_{2}$ and $L$ are known. Assuming the saturated state inside the cup $\left(C_{1}=C_{\mathrm{s}}\right)$ and a zero concentration outside the test specimen $\left(C_{2}=0\right)$, the diffusion coefficient from Eq. 1 is given by:

$$
D=\frac{\dot{m} L}{A C_{s}} .
$$

where $\dot{m}=J \times A$ is the mass flow $(\mathrm{kg} / \mathrm{s})$ of the diffusing VOC, $L$ is the thickness of the material (m), $A$ is the effective area for mass transfer $\left(\mathrm{m}^{2}\right)$ and $C_{s}$ is the concentration of saturated VOC vapour $\left(\mathrm{kg} / \mathrm{m}^{3}\right)$.

The determination of the mass flow through the material can be done experimentally by successive weighing of the diffusion cup. The main disadvantage of this method is that the liquid VOC placed in the cup creates a concentration of saturation, which is unrealistically high for indoor air quality application, with values around dozens of $\mathrm{g} / \mathrm{m}^{3}$ [1]. Indoor concentrations are typically in the order of hundred of $\mu \mathrm{g} / \mathrm{m}^{3}$, achieving values of $\mathrm{mg} / \mathrm{m}^{3}$ in recently built or highly polluted buildings. At low concentrations, diffusion coefficients are generally concentration independent; on the contrary, diffusivities have been shown to vary with concentration at high concentrations [8]. Thus, it is expectable that the wet cup method will overestimate the diffusion coefficient. Haghighat et al. [1] propose the use of the "dry cup method", in opposition to the "wet cup method".

Dry cup method. In the dry cup method, a desiccant e.g., activated carbon, is placed inside the cup to create zero vapour pressure. The test is performed by sealing the test specimen to the open mouth of a cup containing the desiccant and placing the assembly into a controlled atmosphere (Figure 3 ). The test unit is weighed periodically and the weight is plotted as a function of time. In the present work, the desiccant was activated carbon (Sigma Aldrich, 4-12 mesh). 


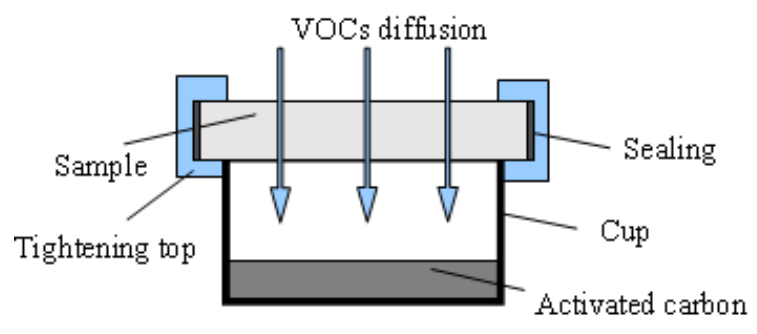

Figure 3. Scheme of the dry cup used for diffusion tests.

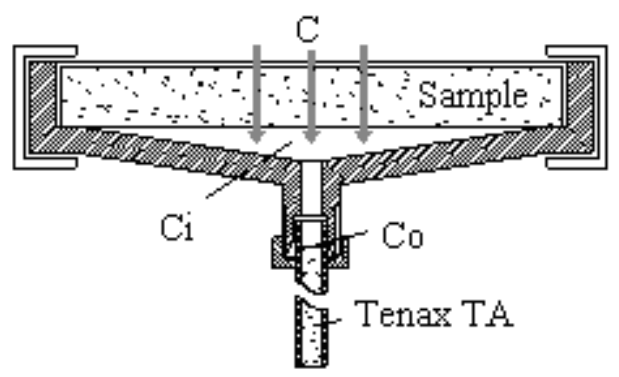

Figure 4 - Scheme of the modified dry cup method used for diffusion tests.

The diffusion coefficient is calculated from the steady-state rate of VOC weight gain, using the Eq. 2, where $C_{s}$ (concentration of saturated VOC vapour) is substituted by $C_{a}$ (concentration of VOC vapour inside chamber). Experiments with the dry cup method have proved to be extremely sensitive to the presence of water vapour, to the point to be as the dominant element in the global transport. It is very difficult to separate the effects of relative humidity in the dry cup method, particularly when the adsorbent has a great affinity for water vapour. In response to this difficulty, it was replaced the method of determining the mass transfer of VOCs, then gravimetric, by an analytical method, by means of passive sampling, where the mass came to be collected by adsorption on Tenax TA tubes and subsequently quantified by gas chromatography - mass spectrometry.

Dry cup method modified. This method can be considered a derivation of the "dry cup", as suggested by the schematic representation of Figure 4. In this method, the tube Tenax TA is connected to the bottom of the cup (modified) and acts as desiccant to adsorb the compounds that diffuse through the material, and then is analyzed by gas chromatography and mass spectrometry (GC-MS). This method allows studying several compounds simultaneously. The passive samplers are based on the mass transport associated with a gradient of concentration that, in dynamic equilibrium, can be expressed by the first law of Fick for diffusion:

$$
\dot{m}=-D A \frac{d C}{d x} \text {. }
$$

Assuming that the adsorbent operates with high capacity for adsorption, we expect a performance in the permanent regime. In these conditions, the flow of VOC can be determined, knowing the mass of the adsorbed compound, $\delta m$, in a time of sampling, $\delta t(\dot{m}=\delta m / \delta t)$.

Assuming negligible resistance to diffusion in the intermediate layer of air, the Eq. 3 for the system represented in Figure 4 can be expressed as:

$$
\dot{m}=\frac{D_{a i r} A_{t}\left(C_{i}-C_{0}\right)}{d} .
$$

where $D_{\text {air }}$ is the coefficient of diffusion of the compound in the air, $A_{t}$ is the area of cross section of sampling tube (Tenax tube), $C_{i}$ is the concentration in the intermediate region (between the material and the sampling tube), $C_{0}$ the concentration of the compound on the surface of the adsorbent and $d$ is the length layer of air. The concentration $C_{i}$ result of diffusion of mass through the porous material between the intermediate region and the environment inside the chamber at concentration $C$. This can be expressed by the equation:

$$
\dot{m}=D_{e} A \frac{\left(C-C_{i}\right)}{L} .
$$


where $D_{e}$ is the coefficient of diffusion effective of material, with area $A$ and thickness $L$.

In passive sampling, is necessary to determine the uptake rate. For a given concentration $C_{i}$, the diffusion uptake rate, $q_{v}$, is given by [9]:

$$
q_{v}=\frac{\dot{m}}{\left(C_{i}-C_{0}\right)}
$$

Ideally, $C_{0}=0$ ("zero sink" conditions). In that case occurs the permanent regime and the uptake rate depends only of diffusion coefficient of the compound in the air and the geometry of the samples used.

$$
q_{v}=\frac{\dot{m}}{C_{i}}=\frac{D_{a i r} A_{t}}{d}
$$

The concentration $C_{i}$, is obtained from the Eq. 7, and $D_{e}$ from the Eq. 5 .

\section{Experimental set-up}

The cup is placed in a test chamber made of stainless steel and with a capacity of $0.255 \mathrm{~m}^{3}$. It is provided with control of temperature and relative humidity. The experimental chamber was cleaned before starting any experiment. All experiments are performed within $(23 \pm 1)^{\circ} \mathrm{C}$ of temperature and $(45 \pm 5) \%$ of relative humidity.

For the diffusion tests, the materials had been cut in form of a circle with diameter of $98.5 \mathrm{~mm}$, carefully in order to maintain the material integrity, and sealed around the edges. For the diffusion experiments three VOCs were chosen with different properties (Cyclohexane, n-Octane and mXylene). The building materials used for testing were as much as possible those that would be used for the actual construction. The experiments were performed with cork parquet without finishing $(\rho$ $\left.=435 \mathrm{~kg} / \mathrm{m}^{3}, L=1.85 \mathrm{~mm}\right)$, gypsum board $\left(\rho=755 \mathrm{~kg} / \mathrm{m}^{3}, L=9.73 \mathrm{~mm}\right)$ and PVC $(\rho=1577$ $\mathrm{kg} / \mathrm{m}^{3}, L=2.07 \mathrm{~mm}$ ). All the samples were placed in a closed box (at $23^{\circ} \mathrm{C}$ and $45 \%$ of $\mathrm{RH}$ ) for stabilization before testing.

A thermal desorption system (from Dani, model SDT 33.50) on line with a gas chromatograph (from Agilent Technologies, model $6890 \mathrm{~N}$ ), coupled to a mass selective detector (from Agilent Technologies, model 5973), for VOC identification and quantification was used.

\section{Results and Discussion}

For both, wet and dry cups, the mass flow rate of VOCs through the material studied in the experiments is given by the slope of the line which reflects the evolution of the mass over time (Figure 5 and 6). The values of diffusion coefficients obtained by the method of "wet cup" are presented in Table 1 and were determined using Eq. 2. The concentration of saturation of the studied compounds obtained in the wet cup method were $273.4 \mathrm{~g} / \mathrm{m}^{3}$ for cyclohexane, $72.1 \mathrm{~g} / \mathrm{m}^{3}$ for octane and $45.5 \mathrm{~g} / \mathrm{m}^{3}$ for xylene. 

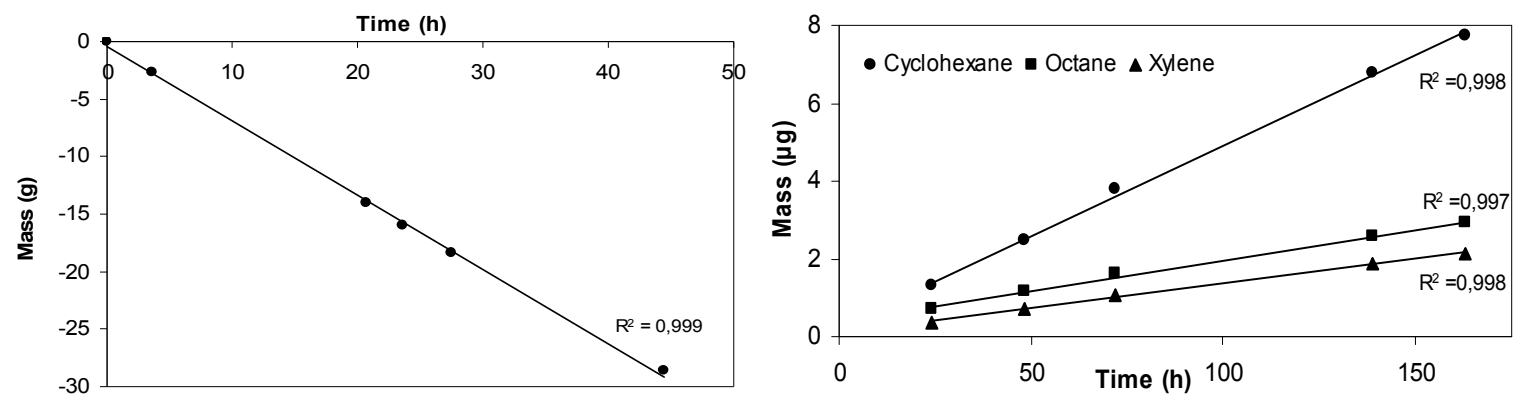

Figure 5. Loss of cyclohexane over time for the gypsum with the wet cup method.

Figure 6. Adsorption of mass by Tenax TA over time for the gypsum with modified dry cup method.

The results show the gypsum board present the higher diffusion coefficients, and the PVC presents the lower $D$. This may be due to the compact structure of PVC, without pores, unlike the gypsum that has a high porosity.

The results of the dry cup method modified were determined using Eq. 5 and are also presented in Table 1 . The concentration of cyclohexane in the chamber been of $5.6 \mathrm{mg} / \mathrm{m}^{3}$, xylene of $4.6 \mathrm{mg} / \mathrm{m}^{3}$ and octane of $4.4 \mathrm{mg} / \mathrm{m}^{3}$.

Table 1 - Experimental values of $D$ obtained by wet cup method and modified dry cup method

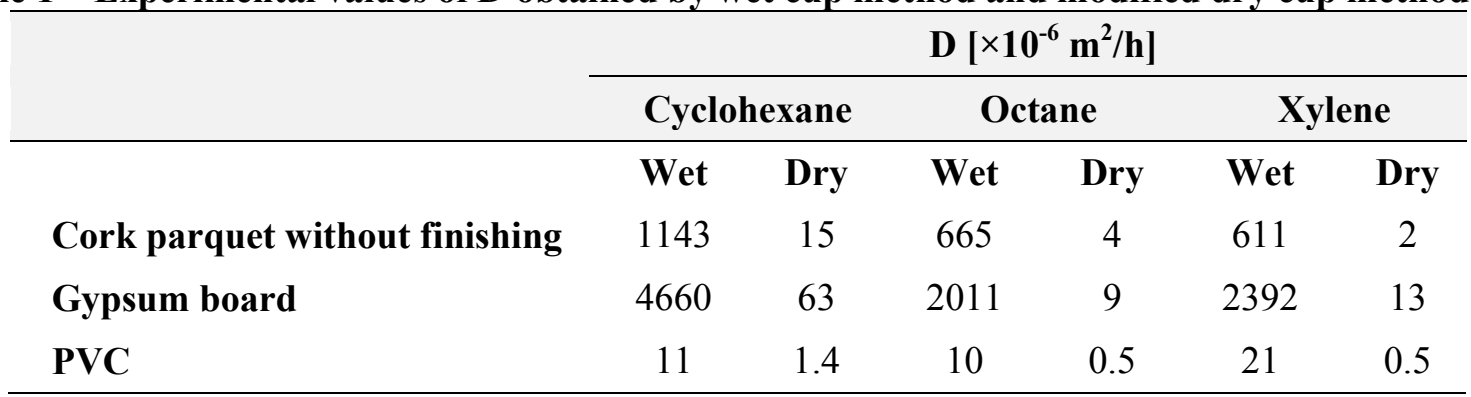

The results obtained by the dry cup method modified are very different compared to the wet cup. Observed differences, varied from the order of $10^{1}$ in the case of PVC to $10^{2}$ in the gypsum and in the cork. It can also be observed that the differences increase with decrease of vapour pressure of the compounds. However some facts were the same: the gypsum board presents the higher values of $D$ and PVC the lower values of $D$ for the three compounds studied in both methods. These differences could be explained by the influence of concentration on diffusion coefficients $[1,8]$, which is very different in the two methods: the saturation concentration in the wet cup method (of the order of hundreds $\mathrm{g} / \mathrm{m}^{3}$ ) and typical concentrations of indoor environment in the modified dry cup method (of the order of several $\mathrm{mg} / \mathrm{m}^{3}$ ).

Moreover, the results obtained experimentally by the modified dry cup method fall within the existing values in the literature, obtained by other methods subject to similar gradients of concentrations, close to the values of indoor environments, like the method of twin chambers [10]. Furthermore, the values obtained by the wet cup method are of the same order of magnitude to those found by other authors who used the same method $[1,7]$.

\section{Conclusions}

The experiments performed by the dry cup method shows it is an extremely sensitive method to the presence of water vapour, being the dominant element in the global transport. It is therefore to highlight the difficulty of separating the effects of relative humidity in the dry cup method, particularly when the adsorbent has a great affinity for water vapour.

In response to this difficulty, it was replaced the method of determining the mass transfer of VOCs, then gravimetric, by an analytical method, by means of passive sampling, where the mass came to be collected by adsorption on Tenax TA tubes and subsequently quantified by gas chromatography - 
mass spectrometry. The results obtained are very much lower compared to the wet cup method, but are in the same range of values published in literature, obtained by different methods, but under similar conditions, as regards to gradients of concentration applied, close to typical values of indoor air.

The differences obtained in the results suggest that the concentration could play an important role in the phenomena of diffusion, affecting the diffusion coefficient obtained experimentally. More tests and data with different materials and compounds will be necessary to confirm this hypothesis, and contribute to the validation of the modified dry cup method.

\section{Acknowledgement}

The authors wish to thank the financial support of FCT for the project "Diffusion of organic vapours in building materials indoors. Influence of the material microstructure'. (PTDC/ EMEMFE/71915/2006).

\section{References}

[1] F. Haghighat, C.S. Lee and W.S. Ghaly: Indoor Air Vol. 12 (2002), p. 81.

[2] A.M. Santos, J.J. Gonçalves, A.O. Martins, G.V. Silva, E.O.Fernandes, Preliminary results on a modified cup method to determine diffusion coefficients of VOCs in building materials. Proceedings of Indoor Air 2008, (2008).

[3] R. Meininghaus, L. Gunnarsen and H.N. Knudsen: Environ. Sci. Technol. Vol. 34 (2000), p. 3101.

[4] P. Blondeau, A.L. Tiffonnet, A. Damian, O. Amiri and J.L. Molina: Indoor Air Vol. 13 (3) (2003), p. 302.

[5] S. Kirchner, J.R. Badey, H.N. Knudsen, R. Meininghaus, D. Quenard, H. Sallee and A. Saarinen: Sorption capacities and diffusion coefficients of indoor surface materials exposed to VOCs: proposal of new test procedures. In: Proceedings of the $8^{\text {th }}$ International Conference on Indoor Air Quality and Climate - Indoor Air 1999, Edinburgh, Vol. 1, pp. 430 - 435, 1999.

[6] R. Meininghaus and E. Uhde: Indoor Air Vol. 12 (2002), p. 215.

[7] M. Guindeira, A. Santos and E. Oliveira Fernandes: Experimental studies on diffusion coefficients of volatile organic compounds for building materials. In: Proceedings of the $10^{\text {th }}$ International Conference on Indoor Air Quality and Climate - Indoor Air 2005, Beijing, p. 1974, 2005.

[8] J. Crank: The Mathematics of Diffusion, Oxford University Press, 1975.

[9] ISO 16017-2:2003 Indoor, ambient and workplace air - Sampling and analysis of volatile organic compounds by sorbent tube/thermal desorption/capillary gas chromatography — Part 2: Diffusive sampling.

[10]C. Richard, C. Neil, J. Fardal, J. Little and X. Ying: Determination of Sorption Parameters for 36 VOC/Material Combinations. Final Report. United States Environmental Protection Agency. 2006. 